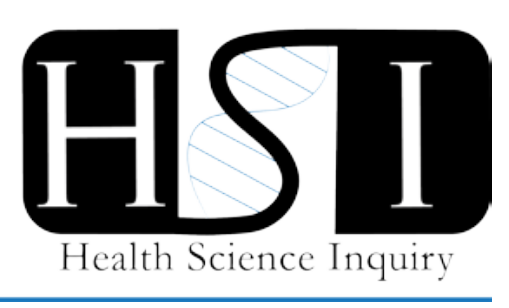

\title{
The genetics of pain: a bio-psycho-social approach for understanding pain
}

\author{
Mohamad Fakhereddin \\ Department of Health and Rehabilitation Sciences, University of Western Ontario
}

Pain is one of the most complex experiences in life due to its subjective nature. Because pain is an intensely personal experience, it is rather difficult to quantify and assess [2]. Most people can judge their own pain but struggle to share the feeling with family, friends, coworkers and health care professionals. This has led to negative stigma directed towards those suffering from chronic pain [1]. As a result, people dealing with chronic pain feel a decrease in effectiveness and productivity and are less motivated to seek treatment [3].

Currently, systematic biology theories indicate pain to be a product of a finely composed balance between the endocrine, immune, and nervous system. However, when one of these systems fail to act, the pain response becomes inharmonious resulting in a chronic problem. Although there is an abundance of literature highlighting the psychological and social significance of chronic pain, understanding how these variables are affected by genetic mechanisms is still in the adolescent stage of research. Biological systems influence cognitive function (and viceversa), making it crucial to study the interactions between biological and psychosocial aspects of pain.

A host of genes have been recognized for their roles in biological systems that respond to distress, trauma, and injury. For the purpose of this commentary, I will focus on a few that are currently emerging as big players in the field of pain and genetics [7]. COMT is a gene responsible for making an enzyme called catechol-O-methyltransferase. The COMT enzyme aids in regulating neurotransmitter levels in the brain by breaking down neurotransmitters such as dopamine and norepinephrine [4]. SLC6A4 encodes the membrane protein responsible for transporting serotonin from synaptic spaces to pre-synaptic neurons. It has been studied for its role in depression-susceptibility in people experiencing emotional trauma [5]. The protein encoded by the FKBP5 gene is a member of the immunophilin protein family. This family of proteins is heavily involved in regulating the immune system, as well as playing a role in basic cellular processes such as protein folding and trafficking [6]. The OPRM1 gene makes a protein known as the $\mathrm{mu}(\mu)$ opioid receptor, which is important for regulating reward, pain, and addictive behaviours in our bodys opioid system [5].

These biomarkers, among others, are important in stress, immune and opioid systems. Moreover, they have been previously studied for their potential involvement in people experiencing acute traumatic injuries. This is primarily done by investigating single nucleotide polymorphisms (SNPs) in these genes. SNPs are mutations in our DNA, represented by a change in a single DNA nucleotide, such as a substitution mutation. The normal occurrence of a SNP results in the most common type of genetic variation among people. Typically, a panel of SNPs can be mapped using a database such as the Human Pain Genetics Database which lists SNPs in human genes that were found to have an association with conditions of pain [7]. Currently, clinical researchers are asking why is it that some people recover successfully after an acute traumatic injury while others do not. A person may have an SLC6A4 or COMT SNP that makes them more psychologically resilient to trauma, thereby enabling them to quickly recover from their injury. Another person may have an OPRM1 SNP that results in lower production of opioid receptors, rendering them unable to properly regulate the opioids they need to manage their pain. Investigating a panel of SNPs in genes that are relevant to trauma and stress to determine whether a relationship exists with psychosocial moderators, such as early life adversity events [8] or household income, will certainly shine some light on pain research. Because genetic research in this particular field is still relatively young, there are many SNPs that still need to be studied alongside psychological and social variables [7]. Hopeful findings are on the horizon which will help both researchers and clinicians better understand pain.

It is well understood that a cascade of physiological changes occurs when a person endures almost any form of pain and the level of these changes are facilitated by genetic factors. Furthermore, these changes are also heavily influenced by psychological and societal contributors such as workplace environment, previous trauma, or other lifestyle stressors [9]. A deeper understanding of this bio-psycho-social model will offer a greater appreciation for the unique pain sensation 
that many people experience. As a result, clinicians and researchers can implement innovative therapeutic strategies based on their ability to empathize with their patients, as well as biological evidence to help solidify rehab programs unique to each persons genetic makeup.

\section{References}

[1] Cohen, M., Quintner, J., Buchanan, D., Nielsen, M., Guy, L. (2011) Stigmatization of patients with chronic pain: the extinction of empathy. Pain medicine, 12 (11), 1637-1643.

[2] Kowalski P. C., Dowben, J. S., Keltner, N. L., (2014) Biological perspectives: pain: it's not all in your head. Perspectives in psychiatric care. 50 (1), 3-6.

[3] Waugh, O. C., Byrne, D. G., Nicholas, M. K. (2014) Internalised Stigma in People Living with Chronic Pain. The journal of pain, 15 (5), 550.e1550.e10.

[4] van Rooij, S. J. H, Stevens, J. S., Ely, T. D., Fani, N., Smith, A. K., Kerley, K. A., Lori, A., Ressler, K. J., Jovanovic, T. (2016) Childhood Trauma and COMT Genotype Interact to Increase Hippocampal Activation in Resilient Individuals. Front. Psychiatry. 7 (1), 156.

[5] Tour, J., Lofgren, M., Mannerkorpi, K., Gerdle, B., Larsson, A., Palstam, A., Bileviciute-Ljungar, I.,
Bjersing, J., Martin, I., Ernberg, M., Schalling, M., Kosek, E. (2017) Gene-to-gene interactions regulate endogenous pain modulation in fibromyalgia patients and healthy controls antagonistic effects between opioid and serotonin-related genes. Pain, 158 (7), 1194-1203.

[6] Ulirsch, J. C., Weaver, M. A., Bortsov, A. V., Soward, A. C., Swor, R. A., Peak, D. A., Jones, J. S., Rathlev N. K., Lee, D. C., Domeier, R. M., Hendry, P. L., McLean, S. A. (2014) No man is an island: living in a disadvantaged neighborhood influences chronic pain development after motor vehicle collision, and this effect is moderated by common genetic variation influencing HPA axis function. Pain. 155 (10), 2116-2123.

[7] Zorina-Lichtenwalter, K., Meloto, C. B., Khoury, S., Diatchenko, L. (2016) Genetic predictors of human chronic pain conditions. Neuroscience. 338 (1), 36-62.

[8] Sadia, S. (2017) A cross-sectional study of stress biomarkers and their associations with post-trauma complaints, and how those associations are moderated by early life adversity. University of Western Ontario Electronic Thesis and Dissertation Repository. 2017; 4856.

[9] Dueñas, M., Ojeda, B., Salazar, A., Mico, J. A., Failde, I. (2016) A review of chronic pain impact on patients, their social environment and the health care system. Journal of Pain Research. 9 (1), 457-467.

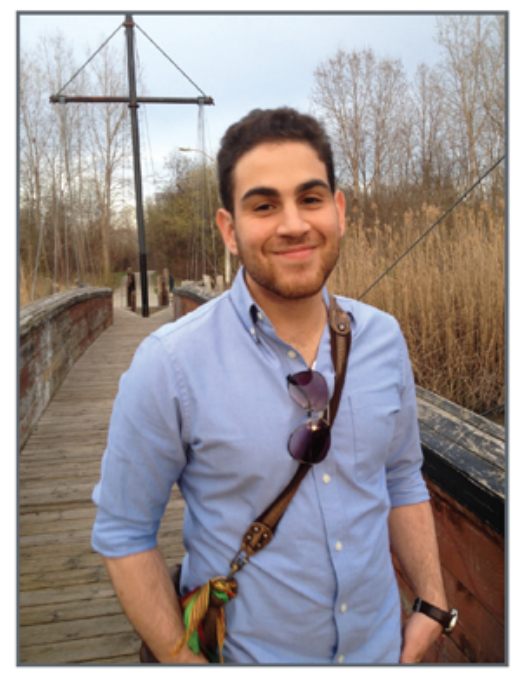

Mohamad completed his Masters in Developmental and Molecular Biology at the University of Windsor. He is now pursuing his PhD with Dr. Dave Walton at Western in Health and Rehabilitation Sciences, specializing in the Physical Therapy stream. Given his extensive molecular research background, Mohamad is interested in looking at genetic polymorphisms in people suffering from acute and chronic pain to further understand the involvement of biological mechanisms in musculoskeletal pain and trauma. His work is part of a much bigger project known as the SYMBIOME Project, taking place in the Pain and Quality of Life Integrative Research Lab (PIRL). 